\title{
Remarques sur l'Aselle psammique Proasellus walteri (Chappuis, 1948) (Crustacea, Isopoda, Asellota)
}

\author{
par \\ Jean-Paul HENRY*
}

\begin{abstract}
SUMMARY
Remarks about the psammic Asellid Proasellus walteri (Chappuis, 1948) (Crustacea, Isopoda, Asellota).
\end{abstract}

$P$. walteri, an eyeless species of tiny size and thin body, shows numerous original characters. By its general morphology, it is one form of Asellid best adapted to the phreatic waters where it exists in large settlements. It is also able to live in the psammic biotope in a manner similar to Microparasellids. The females lay only 6 to 10 normal sized eggs. There is no indication of oostegits outside the breeding period. In some populations, the small number of eggs, correlated with the small size, seems to be compensated by a sex-ratio favorable to the females.

La récente révision systématique de l'ancienne espèce anophtalme Proasellus cavaticus (Henry 1971, 1973) devait conduire à scinder cette forme polytypique en quatre espèces distinctes: $P$. cavaticus, $P$. valdensis, $P$. strouhali et $P$. walteri. La comparaison de ces quatre formes hypogées met en évidence les particularités de $P$. walteri qui apparaît comme l'une des espèceses les plus originales du genre Proasellus.

Considéré à l'origine comme une simple sous-espèce de cavaticus, cet Aselle fut décrit assez sommairement à partir d'une récolte de quelques individus dans la nappe alluviale d'un petit affluent jurassien du Rhin aux environs de Bâle (Chappuis 1948). Pendant plus de vingt ans aucune nouvelle station ne fut découverte et $P$. walteri resta très peu connu parmi la faune d'Asellides. Depuis 1969 de nombreuses stations furent mises en évidence et permirent de reconnaître l'indépendance spécifique de cette forme. C'est pourquoi il est intéressant d'attirer l'attention sur son originalité morphologique en donnant une description plus détaillée. $P$. walteri est une très petite forme, à corps grêle et fili-

\footnotetext{
* Laboratoire de Biologie Animale et Générale, Faculté des Sciences de la Vie, Université de Dijon,
} 6 bd Gabriel, 21000 Dijon, France. 


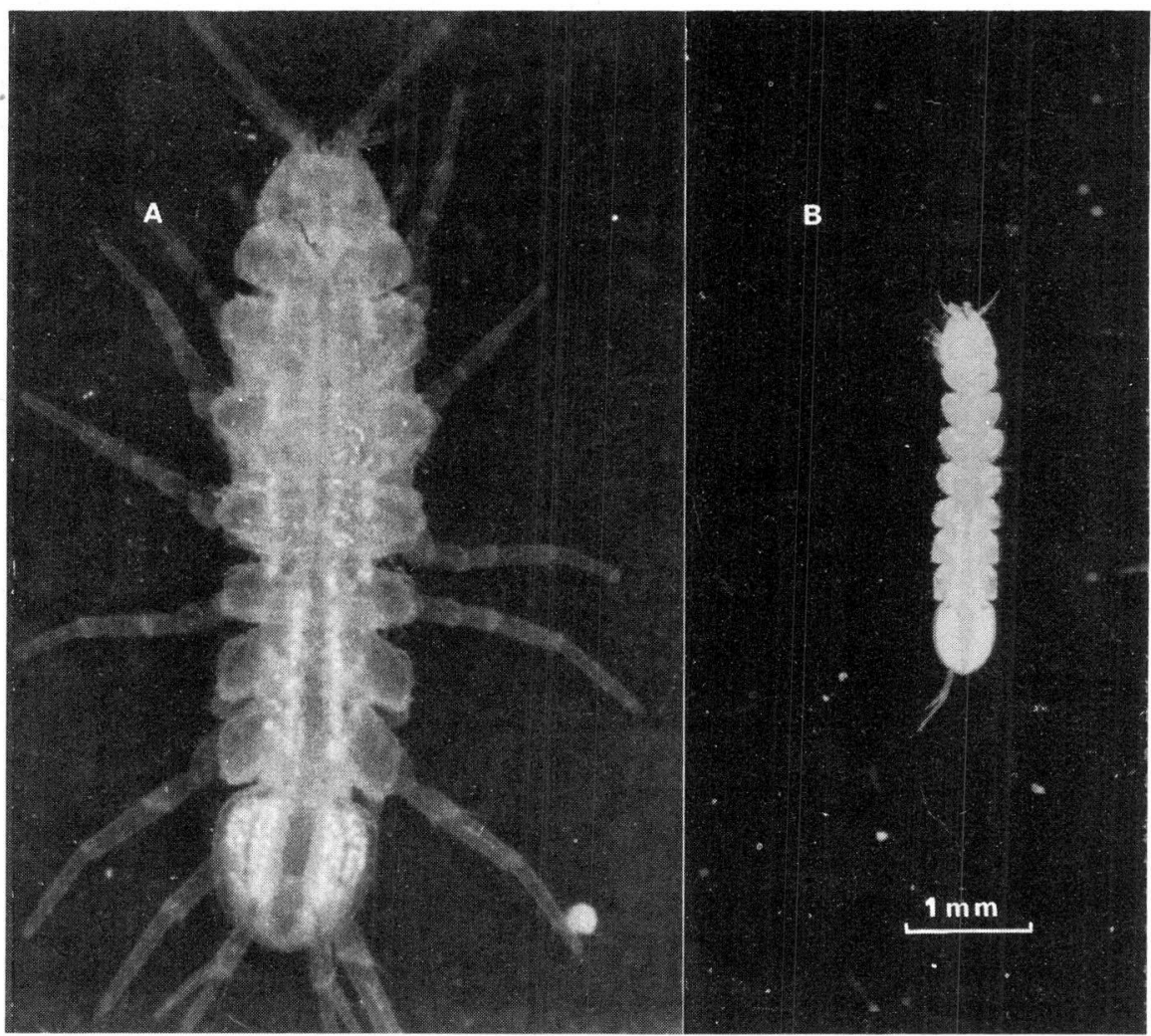

Pl. I: Comparaison de deux espèces d'Aselles des eaux souterraines

- A - Proasellus cavaticus, forme anophtalme fréquente dans les eaux libres des grottes, mais capable également d'envahir le domaine interstitiel à gros interstices.

B - Proasellus walteri, forme exclusivement interstitielle, parfaitement adaptée à ce mode de vie; sa petite taille lui permet de peupler aussi le milieu psammique.

Fig. 1: Proasellus walteri (Chappuis, 1948) car. emend. Henry 1971.
A: vue tergale d'un mâle de $2,5 \mathrm{~mm}, \mathrm{e}=0,4 \mathrm{~mm}$.
B: pléopode I $\hat{o}, \mathrm{e}=50 \mu$.
C: pléopode II $\hat{\delta}, \mathrm{e}=50 \mu$.
D: région distale de l'endopodite du pléopode II $\hat{O}, \mathrm{e}=100 \mu$.
E: pléopode II $O, \mathrm{e}=50 \mu$.
F: pléopode III des deux sexes, e $=100 \mu$.
G: pléopode IV des deux sexes, e $=100 \mu$.
$\mathrm{H}$ : pléopode $\mathrm{V}$ des deux sexes, $\mathrm{e}=100 \mu$.
I: extrémité de l'antennule, $\mathrm{e}=50 \mu$.
$\mathrm{J}$ : maxillipède d'une femelle ovigère, $\mathrm{e}=100 \mu$.
$\mathrm{K}$ : péréiopode III, e $=100 \mu$.
L: uropode gauche, $\mathrm{e}=100 \mu$. 
REMARQUES SUR PROASELLUS WALTERI

77

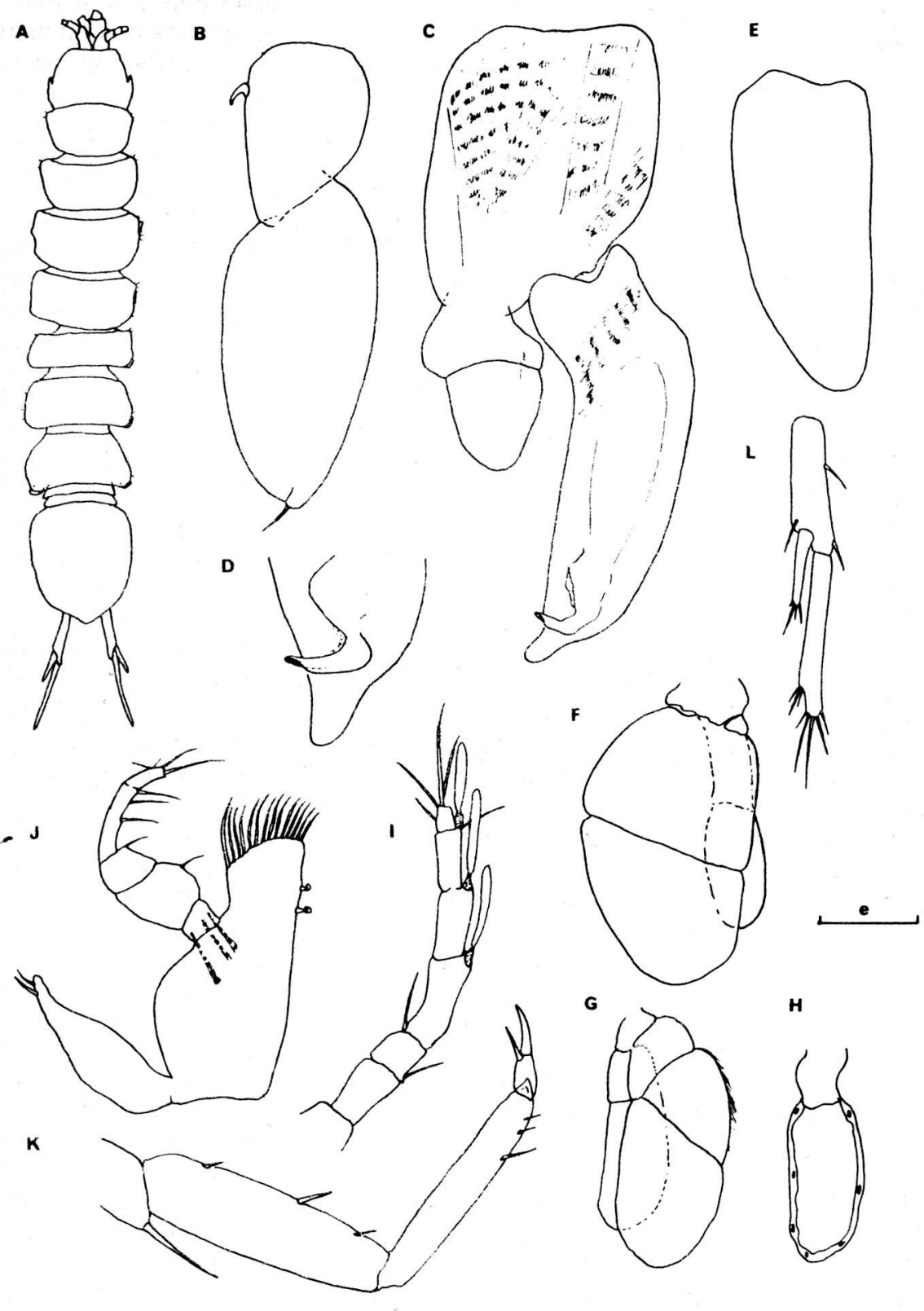


forme dont le coefficient d'allongement est voisin de 6; les plus grands individus dépassent rarement $2,5 \mathrm{~mm}$, c'est dire que la taille de cette espèce est nettement inférieure à celles des autres Proasellus $(\mathrm{Pl}$. I et fig. 1 A). Son caractère le plus remarquable est l'aspect de son pléopode II ô (fig. 1C) dont l'endopodite est presque trois fois plus long que l'exopodite, alors que sa région distale présente une structure caractéristique du groupe cavaticus. Parmi les caractères distinctifs de $P$. walteri, on notera aussi une chétotaxie très réduite sur l'ensemble des appendices; c'est ainsi que le pléopode I $\hat{\delta}$ ne porte qu'une seule soie au bord apical, à côté d'une petite encoche inhabituelle sur cette rame; le pléopode II $q$ est parfaitement glabre et les autres pléopodes ne présentent aucune soie marginale. Cette réduction du nombre des phanères se retrouve également sur les péréiopodes qui sont extrêmement grêles et ne portent qu'une seule épine sur leur dactylopodite (fig. 1K). Les uropodes sont très grands et sont aussi longs que le pléotelson, avec un exopodite nettement plus court que l'endopodite. Ces appendices, ainsi d'ailleurs que les antennes et les péréiopodes, sont d'une fragilité extrême ce qui explique que lors des récoltes les individus soient pratiquement tous mutilés. Enfin, parmi les caractères originaux, on peut encore citer les maxillipèdes des femelles ovigères qui ne portent aucune trace d'oostégite, ce qui est exceptionnel chez les Asellides et la présence de pléopodes $\mathrm{V}$ uniramés.

La morphologie générale de cette espèce montre sans conteste qu'elle est une des formes d'Asellides les mieux adaptées à vivre dans le milieu interstitiel. D'ailleurs, à ce jour, elle n'a jamais été mise en évidence dans les eaux libres des grottes. Considéré pendant longtemps comme très rare et localisé, $P$. walteri apparaît au contraire, actuellement, grâce à l'efficacité des méthodes de récoltes (sondages tubés) comme un hôte permanent et abondant des nappes alluviales du bassin du Rhône. Dans ce domaine interstitiel, les peuplements de $P$. walteri sont particulièrement bien développés, tant par leur densité que par leur étendue, dans le flux souterrain des rivières, où l'espèce cohabite parfois avec des Aselles de plus grande taille, oculés comme $P$. meridianus, ou anophtalmes comme $P$. cavaticus et $P$. strouhali. Ce dernier type d'association a été un argument en faveur de l'hétérogénéité spécifique de l'ancien $P$. cavaticus; en effet, si deux formes vivent ensemble sans s'interpénétrer, c'est qu'elles appartiennent chacune à une espèce distincte. Dans ces cohabitations, la répartition des individus doit d'ailleurs être en rapport avec la granulométrie des alluvions; ainsi il faut imaginer que les walteri se trouvent entre les éléments les plus fins, alors que les cavaticus ou les strouhali vivent dans les plus gros interstices. Mais c'est sa présence dans le milieu psammique qui constitue la caractéristique écologique la plus remarquable de $P$. walteri. En effet, en Bourgogne, cet Aselle forme de larges et denses peuplements dans des nappes phréatiques superficielles contenues dans des dépôts quaternaires récents représentés par des sables très fins et des limons calcaires. Ce type d'habitat diffère nettement des autres biotopes interstitiels tant par la granulométrie de ses éléments que par son peuplement. $P$. walteri, dont la taille est en harmonie avec les dimensions réduites des interstices, est probablement un des rares Asellides à être capable de vivre dans un tel milieu. On le trouve ainsi en compagnie d'animaux 
inféodés à ce biotope, et en particulier avec les Bathynelles. Cette faculté de vivre dans le milieu psammique amène à comparer $P$. walteri aux espèces de Microparasellides du genre Microcharon qui occupent des biotopes analogues dans les eaux interstitielles continentales des régions plus méridionales. Les quelques exemplaires de walteri maintenus en élevage m'ont permis d'observer que cette forme évolue entre les grains de sable avec une grande agilité, comme ces Isopodes interstitiels, et se montre également capable de marcher à reculons avec une vitesse extraordinaire. La concentration de $P$. walteri dans ce biotope est surprenante: lors d'une première récolte au cours de laquelle 120 litres d'eau ont été filtrés, 360 individus furent recueillis, alors que le lendemain au même endroit, le même volume d'eau permettait d'en dénombrer plus de 400 . Un mois après ces prélèvements, de nouveaux sondages montraient que la densité de la population était toujours aussi forte. On peut penser que l'absence de la macrofaune interstitielle, notamment celle des prédateurs comme les Niphargus, favorise le développement de cette espèce dans ce milieu souterrain.

Les données biologiques sur $P$. walteri, bien qu'elles soient encore fragmentaires, sont également très intéressantes car elles permettent de mieux caractériser l'espèce. L'observation de femelles ovigères montre que le nombre d'oeufs contenus dans le marsupium est très faible: il est de 6 en moyenne et semble ne pas dépasser une dizaine chez les plus grandes femelles. Cet effectif est très réduit par rapport à celui des Aselles épigés et même hypogés. $P$. $m e$ ridianus pond entre 10 et 100 oeufs, et $P$. cavaticus entre 10 et 70 . Pour ces dernières formes, il existe, comme c'est la régle chez les Péracarides, un lien étroit entre le nombre des oeufs et la taille de la femelle; en effet, l'effectif de la portée d'une femelle est directement fonction du volume de sa poche incubatrice, dont les dimensions sont proportionnelles à celles du corps de la femelle. On comprend alors que les portées de $P$. walteri, dont le corps est presque trois fois moins long et moins large que celui des deux autres Proaselles, ne comportent qu'un petit nombre d'oeufs. Cette diminution du nombre des oeufs est d'ailleurs un phénomène général chez tous les animaux interstitiels et en particulier chez les Isopodes; on la considére le plus souvent comme un caractère lié au mode de vie particulier de ces espèces. Or, il semble bien qu'elle soit, avant tout, corrélative de leur faible taille. L'oeuf de $P$. walteri est de taille comparable à celui des autres espèces de Proasellus: il mesure environ $250 \mu$ sur $280 \mu$ alors que les oeufs des formes épigées ou hypogées atteignent 300 à $320 \mu$. On n'observe donc pas chez cet Aselle d'augmentation de la taille des oeufs qui pourrait expliquer en partie la réduction de leur nombre, comme c'est le cas chez d'autres Isopodes interstitiels. Bien entendu, en comparaison des dimensions de la femelle, les oeufs paraissent relativement gros. Cette remarque doit s'appliquer aux autres espèces interstitielles réputées pour la taille élevée de leurs oeufs, car il semble bien que la taille de l'oeuf soit assez constante dans chaque groupe d'Isopode.

Comme chez tous les Asellides, les jeunes quittent la poche incubatrice à l'état larvaire, puis subissent plusieurs mues dans les mois suivants pour acquérir leur morphologie d'adulte. Les caractères de ces diférents stades seront précisés prochainement dans le cadre d'une étude détaillée du développement 
post-embryonnaire de $P$. cavaticus. Quelques jours après la libération des larves, la femelle subit une mue particulière au cours de laquelle elle perd sa poche incubatrice. Mais, ici, contrairement à ce qui se passe chez les femelles d'Asellides, il ne subsiste pas d'oostégites de taille réduite: la femelle passe directement lors de la mue parturielle du stade sans oostégite au stade à grands oostégites fonctionnels. Ainsi les oostégites ne sont, chez $P$. walteri, qu'un caractère sexuel temporaire qui ne se manifeste qu'au moment de l'incubation; le phénomène qui s'observe aussi chez certaines espèces de Microparasellides semble donc caractériser les formes interstitielles.

Si le nombre d'oeufs pondus par $P$. walteri est assez faible, il ne pose pourtant aucun problème démographique à l'espèce à en juger par l'abondance des individus récoltés. Pour certaines populations, j’ai observé une nette prédominance du nombre des femelles par rapport à celui des mâles; en effet, si en général, la proportion des sexes est légèrement en faveur des femelles chez les Aselles, le pourcentage des femelles peut atteindre chez $P$. walteri plus de $80 \%$. Un taux aussi élevé de femelles permet donc d'atténuer en partie la prolificité réduite de cette espèce.

Avec cet Aselle anophtalme, dont tous les caractères traduisent une parfaite adaptation à la vie psammique, la conception classique que les Aselles hypogés sont des formes essentiellement cavernicoles devient inexacte. Il faut, en effet, désormais tenir compte de ce que certains Asellides, tel $P$. walteri, constituent un élément colonisateur important du domaine interstitiel.

\section{RÉSUMÉ}

$P$. walteri est une espèce anophtalme de très petite taille et à corps grêle et filiforme qui possède un ensemble de caractères originaux. Sa morphologie générale montre qu'elle est une des formes d'Asellides les mieux adaptées à coloniser le domaine interstitiel où elle constitue d'importants peuplements. Elle est aussi capable de vivre dans le milieu psammique où son comportement rappelle celui des Microparasellides. La femelle de $P$. walteri ne pond que 6 à 10 oeufs de taille normale; elle ne porte aucun oostégite, même de taille réduite, en dehors des périodes d'incubation. La diminution du nombre des oeufs pondus, corrélative de la faible taille de l'espèce, semble être atténuée, dans certaines populations par une sex-ratio nettement en faveur des femelles.

\section{BIBLIOGRAPHIE}

CHAPPUIS, P. A., 1948: Copépodes, Syncarides et Isopodes des eaux phréatiques de Suisse. Rev. Suisse de Zool., 55, 549-566.

HENRY, J.-P., 1971: Contribution à l'étude du genre Proasellus: le groupe cavaticus. Vie Milieu, Paris, 22, 33-77.

HENRY, J.-P., 1973: Contribution à l'étude du genre Proasellus: l'espèce cavaticus. $6^{\circ}$ Cong. Intern. Spéléol. Olomouc, sous presse. 